\title{
In defence of taxonomy
}

In Nature of 16 August (Vol. 346, 602; 1990), H. T. Clifford, R. W. Rogers and M. E. Dettmann argued that taxonomists might usefully dispense with the existing massive herbarium collections. We have received many letters criticizing this view. Here we publish those received first; the remainder make many of the points below.

SIR-Clifford, Rogers and Dettmann have exaggerated the problems faced by taxonomic institutes and have misunderstood the role of herbaria. They suggest a solution that displays ignorance and a surprising lack of understanding in professional biologists.

The worry is that their solution may appeal to the uninitiated, and could be taken up by busy administrators and politicians seeking quick remedies to immediate ills. As representatives of the systematic botany community in Australia, we would like to stress that systematics is not merely an exercise in stamp collecting or a naming service for other branches of biology.

Briefly, Clifford et al. state that herbaria are becoming choked by everincreasing numbers of specimens, most of which, in their opinion, have so little value that we would be better off without them; they should, the authors say, be pulped. The principle can be applied to all taxonomic collections. With touching optimism, they go on to suggest that funds and staff-time so saved would be diverted to "taxonomic research proper".

No part of Australia's flora is well known in toto, but we probably all know of individual species that are so wellrepresented that some specimens could be pruned without loss. But even if a specialist were to prune, the saving in curatorial load would be negligible. All the institutes we represent already practise some pruning and quality control of incoming material; some reserve sterile material apart until after publication of results, and disposal seems appropriate. But no case at all can be made for ditching the bulk of the collections.

The lack of understanding of the difference between written records and specimens shown by Clifford et al. is little short of stupefying. A description makes accessible a selection, a subset, of the total information that a specimen yields. There is no such thing as a complete description; there will be as many descriptions as there are disciplines studying that specimen, and many of them will not overlap. Yesterday we would have had descriptions of gross morphology, anatomy and palynology. Today, we have electron microscopy and biochemistry in many new and revealing facets. Tomorrow, who knows? No specimens, no information.

Today, systematic biology is being rejuvenated by new and more disciplined ways of thinking; the computer provides powerful new tools and the predictive power of the resulting classifications the central aim of the systematist improved. Without specimens, variation cannot be assessed. The amassed collections of ourselves and our forebears now have new potential in the urgent task of discovering, describing, naming and above all understanding the relationships and biology of the riot of life around us while it lasts. It is time to build on the resources of our collections, not to discard them untapped.

Finally, the authors confuse herbaria (the collection of dried plant specimens) with Herbaria (the institutes that care for and use them). The value and usefulness of herbaria are judged by the number of specimens, the geographical areas covered, the groups represented, the state of their curation and the proportion of 'classical' material mentioned in the literature, including types. But Herbaria are indeed judged in part by the quality of their research, in part by their attitude and accessibility to visiting researchers. The quality of the research is a much more complex mix of factors than Clifford et al. allow - published floras, monographs and papers in the scientific literature are the most obvious, but accuracy in the identification of collections derives in large part from long familiarity with the collections. In turn, these identifications are the key to the literature and are thus of crucial importance to all those other disciplines that rely on taxonomists' insight and experience.

It is to be hoped that those concerned with support and management of our biological collections are not misled by the simplistic, short-sighted and ill-conceived ideas put forward by Clifford et al. Comprehensive, well-curated collections are essential for the production of highquality systematic research sought by these authors

Division of Plant Industry,

CSIRO, PO BOX 1600,

Canberra, ACT 2601, Australia

BARRY J. CONN

Royal Botanic Gardens,

Sydney, NSW 2000, Australia

SIR-Clifford et al. raise some interesting points concerning housing collections in the natural sciences. But I will not be jettisoning any part of our herbarium.

First, plants, unlike chemical compounds, are much more complex subjects where any current description interprets only $x$ out of $n$ characteristics. Experience shows that the next investigator will want to see all the available material, not just an historical account

Second, we keep well-documented specimens, as well as type(s), because the originally described material may not adequately show the range of variation in species (super-orders are too coarse a taxonomic category) and may not reflect the changing distribution of species.

Finally, reference material is needed to identify enquiries and for educational purposes, and specimens are both historically interesting and aesthetically pleasing.

At a time when many institutions in the public sector are under pressure to provide short-term solutions to cash problems, it is tempting to cast doubt on long-term scientific objectives and commitments to material culture. But with most of the world's species still to be documented, this is no time to weaken our resolve. Rather, natural scientists should focus their skills and what resources they have left in overcoming the mid-term blues. They could start with a look at collecting policies and regional needs.

E. A. JARZEMBOWSKI

Booth Museum of Natural History, 194 Dyke Road, Brighton BN1 5AA, UK

SIR-The taxonomic Brave New World outlined by Clifford et al. is strange indeed: natural history collections, they say, are not needed because chemists do not store the compounds they synthesize; taxonomy should be based on descriptions and type specimens and should have a rational economic basis.

But one cannot synthesize individuals as a chemist synthesizes compounds. A chemical compound and a herbarium sheet are not comparable entities, as philosophers of science have long acknowledged. To say they differ in degree, not nature, simply will not do.

Descriptions alone, even when accompanied by type specimens, are no basis for the comparative biology of the future. For one thing, descriptions are often poor representations of what is described and, in this area, taxonomists need to progress. And taxonomists make mistakes.

By way of illustration, species limits now need extensive change in a phylogenetically critical group related to the mangosteen (Garcinia mangostana), found from the Philippines to New Caledonia. I know this only because I have access to collections assembled at great cost over 250 years; the descriptions of the species, even recent descriptions, are of little help, but the specimens, and some new characters, are.

Knowledge of plants in the field is important, but well-maintained herbarium collections contain a mine of information. Future monographers are 
likely to rely more, rather than less, on herbarium collections for an understanding of some aspects of variation patterns. These collections will more and more represent populations that have become extinct because of man's activities.

It is ironic that a photograph of Kew Herbarium should accompany the article by Clifford et al. Almost 100 years ago, a director of Kew, W. T. Thistleton-Dyer, suggested a similar course of action to the one they propose. He accepted variation, but thought only a single specimen of each species, representing the typical morphology of the species, was needed in the herbarium. A number of 'duplicates' were removed from the collection at Kew, and some sent to Berlin - where they were described as new species (B. Verdcourt, personal communication).

Natural history collections are a celebration of diversity, right down to the level below the species, but Clifford et al. rightly ask whether we need all the collections. Problems attendant on the storage of voucher specimens are real. Clifford et al. might also have noted that many duplicates of one genotype may be taken from a single tree, and that 20 duplicates housed in as many herbaria might be excessive, useful though they may be in evaluating variability.

One wonders if the general solution proposed by Clifford et al. is a justification for cutting down on herbarium activities in a particular university department; a rationalization after an all-too-common academic tragedy. Being associated both with a large herbarium and a university department, I believe that rather different kinds of research may be appropriate for the two. But the proposals made by Clifford et al. will help neither academic institutions nor the herbaria; taxonomy must first have a rational scientific basis, and to destroy the collections will not provide this.

P. F. STEVENS

Hanvard University Herbaria, Cambridge,

Massachusetts 02138, USA

SIR-I was surprised that three biologists, no doubt experts in their own fields, should appear to have such naive views about the value and methodology of basic taxonomy as to suggest that our collections should be turned over to the garbage collectors. Even a superficial consideration reveals that their suggestion is completely counter to the rigorous methods entailed in scientific research, which should ensure that results are based on repeatable analyses of natural phenomena.

Clifford et al. say "Biological specimens may be different from [chemical specimens], but they differ only in degree not in kind". A chemical element or compound can be rigidly defined and shows variation only within certain repeatable limits, whereas a biological species is of necessity defined according to one type specimen of a species, a species being composed of many individuals showing a range of variation. It is impossible to express the nature and extent of this variation in its entirety and it is therefore necessary to sample it and to keep material for research. Chemicals do not reproduce, neither do they pass on heritable characteristics to their offspring. The destruction of herbarium material, as suggested in the Commentary, would ensure that no one could then verify or refute results which did not appear to agree with the main body of data about, say, a species.

One of the more grotesque suggestions of Clifford et al. is that decisions on taxonomic changes should be based solely on published descriptions. Perhaps the best way to show the fallacy of their argument is to consider one particular case.

Epling prepared a revision of the genus Eriope from South America in 1936. He was a careful and productive worker, but in this case his research had many defects. $\mathrm{He}$ recognized 21 species. If all the material had subsequently been destroyed, I would not have been able to revise the genus, finding that one species had been misinterpreted and belonged to another genus and another had been misinterpreted and belonged to a different family. Of the remaining nineteen, one was better placed in a separate genus, due to characters which Epling had failed to observe, and a further six I was able to reject as one species, as the result of careful analysis of the range in variation of the other material of these taxa available in the form of herbarium data, which Clifford et al. would have pulped. I also described a further three new species using material sent to me by other herbaria.

My publication on this topic, which included distribution maps to show biogeographical patterns, would have been impossible without all the herbarium material at my disposal, including not only new but also re-identified materials wrongly assigned by Epling.

As a result of my studies on Eriope and related genera, I was able to regroup the taxa into genera that more closely reflected their natural relationships. Nevertheless, my fieldwork has also shown me that I am not infallible, but that some opinions I held at the time of my 1976 revision were erroneous. Thank goodness the herbarium material is there to prove it.

To prepare a data-matrix for cladistic or numerical analysis, it is always necessary to re-investigate characters that may have been ignored or misinterpreted by earlier workers. This would be impossible in the taxonomic wasteland proposed by Clifford et al., or perhaps these authors do not believe in such methods.

Finally, because there is at present a shortage of funding in the sciences, it is fair and proper to look at which areas need greatest priority of support. But, under these circumstances, I am suspicious of those who, through ignorance, are prepared to back the destruction of a branch of science other than their own. There is, of course, frustration that the classification and description of the approximately 200,000 species of flowering plant is still far from complete. Today, the need for this is ever more urgent, as pressure on natural environments becomes more acute. The fault, however, is not due to the lack of efficiency nor lack of desire to use modern methods, but is due to lack of resources. Science and industry rely on taxonomists, but there is less willingness to acknowledge that the quality of our expertise is inevitably related to resources put at its disposal.

Royal Botanic Gardens, Kew, Richmond, Surrey TW9 3AB, UK

SIR-There is a gross mischief about that taxonomic research is unrelated to the curation, maintenance and expansion of herbarium collections. It has even been suggested by Clifford et al. that it is not necessary to maintain large collections of plant specimens in herbaria, but instead that most collections can be pulped, replacing them with computerized label data. Such a radical proposal may seem sensible and plausible to many of Nature's readers, but it is based on unsound logic and a less than full appreciation of taxonomy as a science. Researchers from other fields should know why we are so upset and should appreciate how the proposals put forward by Clifford et al. could eventually affect them.

We have all spent time in European herbaria either because our countries did not have adequately named herbarium material, or because our herbaria lacked types and literature. It was only in these large centres that we could complete our work or find solutions to taxonomic problems raised by our colleagues.

Clifford et al. suggest that there is no need to collect representative specimens of plants from different localities, at different times of the year, at different stages of the life cycle, of different ecotypes, with different chemical constitution, and so on, and say that it is wasteful to keep voucher specimens for biological and chemical research. Their suggestions would lead to the destruction of the only validating evidence that the specimen(s) used in experiments was correctly identified. For instance, one of us (C.H.S.) recently collected the few surviving fragments of seeds of sophoroid legumes used by chemists to isolate new chemical compounds, only to find that some of the species were not Sophoreae and did not even belong in the family Leguminosae. There were no 